\title{
CAYLEY GRAPHS VERSUS ALGEBRAIC GRAPHS
}

\author{
Pranjali $^{1}$, Amit Kumar ${ }^{2}$, And TANuja YadaV $^{3}$ \\ ${ }^{1}$ Department of Mathematics, \\ University of Rajasthan, JLN Marg, Jaipur 302004, India, \\ pranjali48@gmail.com \\ ${ }^{2,3}$ Department of Mathematics and Statistics, \\ Banasthali Vidyapith, Banasthali-304022, India, \\ amitsu48@gmail.com, yadav.tanuja17@gmail.com
}

\begin{abstract}
Let $\Gamma$ be a finite group and let $S \subseteq \Gamma$ be a subset. The Cayley graph, denoted by $\operatorname{Cay}(\Gamma, S)$ has vertex set $\Gamma$ and two distinct vertices $x, y \in \Gamma$ are joined by a directed edge from $x$ to $y$ if and only if there exists $s \in S$ such that $x=s y$. In this manuscript, we characterize the generating sets $S$ for which $\operatorname{Cay}(\Gamma, S)$ is isomorphic to some algebraic graphs, namely, unit graphs, co-unit graphs, total graph and co-total graphs.

Key words and Phrases: Cayley Graphs; Finite group, Generating sets.
\end{abstract}

\section{Preamble}

The research in algebraic combinatorics is aims at exposing the relationship between algebra and graph theory and at advancing applications of one to the other. In the last decade, many authors have studied algebraic graphs(Viz., zero-divisor graph, total graph, unit graph, co-total graph, maximal graph, co-unit graphs, prime graph, etc) associated to algebraic structures. Perhaps the best known one is the Cayley graph [2] of a group.

Let $\Gamma$ be an abelian group. For $\mathbb{Z}_{n}$, the group of integer modulo $n$, the sets $Z\left(\mathbb{Z}_{n}\right)$ and $U\left(\mathbb{Z}_{n}\right)$ are defined as; $U\left(\mathbb{Z}_{n}\right)=\{x: \operatorname{gcd}(x, n)=1\}$ and $Z\left(\mathbb{Z}_{n}\right)=\{y$ : $\operatorname{gcd}(y, n) \neq 1\}$. For generating set $S$ of $\Gamma$ we define the Cayley graph of $\Gamma$ to be the (simple) graph $\operatorname{Cay}(\Gamma, S)$ with vertices $\Gamma$, the set of elements of $\Gamma$, and two distinct vertices $x$ and $y$ are adjacent if and only if there exists $s \in S$ such that $x=s y$. Thus $\operatorname{Cay}(\Gamma, S)$ is connected graph when $S \subseteq U(\Gamma)$, and $\operatorname{Cay}(\Gamma, S)$ is disconnected

2020 Mathematics Subject Classification: 13A15, 05C22, 05C75, $16 \mathrm{P} 10$.

Received: 22-01-2019, accepted: 05-04-2021. 
when $S \subseteq Z(\Gamma)$. With this motivation in Section 2, we characterize the generating set $S$ for which $\operatorname{Cay}(\Gamma, S)$ is isomorphic to one of the graph, namely, unit graphs, co-unit graphs, total graph and co-total graphs. However, for convenience of the reader, we shall also gather some definitions and results for Cayley graphs which will be used in the sequel.

In Section 3, we give several basic results about Cayley graphs associated with some specific generating sets. We have also established the results that gives the relation between Cayley graph and unit graphs, co-unit graphs, total graph and co-total graphs. Further, in view of Theorem 3.3 we have shown that the study on the notion of unit graph, co-unit graphs, co-total graphs and total graph for the group $\mathbb{Z}_{n}$ is a particular case for the study on the $\operatorname{Cay}\left(\mathbb{Z}_{n}, S\right)$ associated with specify generating sets, viz., $U\left(\mathbb{Z}_{n}\right)$ and $Z^{0}\left(\mathbb{Z}_{n}\right)$. Throughout, many examples are given to illustrate the theory, and we pose several questions. For detail study about co-total graph, and co-unit graphs, we refers to reader [5].

In what follows, all consider graphs are simple, i.e., undirected graphs in which any two vertices are joined by at most one edge and without loops. Throughout, $\mathbb{Z}_{n}$ will denote the group of integers modulo $n$; and $C_{n}, K_{n}$, and will denote an $n$-cycle, and the complete graph on $n$ vertices, respectively. To avoid trivialities, we implicitly assume when necessary that graphs are nonempty. For terminology and notation from group theory or graph theory not defined in this paper, we refer the reader to [4] and [3], respectively.

\section{Generating Sets}

In general, given any group $\Gamma$, a generating set $S$ of $\Gamma$ is merely a nonempty set such that $e \notin S$ and if $a \in S$, then $a^{-1} \in S$, where ' $e$ ' is the identity element of $\Gamma$. Consequently, $S \neq \phi$ and if all the elements of $\Gamma$ are self inverse, then all possible generating sets are $2^{n}-2$. In view of this definition, the following result recognize the structure of such generating set. In 2011, Beny and Rakhmonov [1] have derived a formula for number of Cayley graphs on $\mathbb{Z}_{n}$.

Theorem 2.1. Let $\Gamma \cong \mathbb{Z}_{n}$ and $S$ be a generating set of $\Gamma$ and $N(S)$ be the collection of all generating sets. Then

i) If $n$ is odd, then $|N(S)|=2^{\frac{n-1}{2}}-1$;

ii) If $n$ is even, then $|N(S)|=2^{\frac{n}{2}}-1$.

Example 2.2. Consider the group $\mathbb{Z}_{4}$, then there are precisely three generating sets $\{1,3\},\{2\}$ and $\{1,2,3\}$. If we take $n=4$ in Theorem 2.1, then we get $|N(S)|=3$. In the similar vein, consider the group $\mathbb{Z}_{5}$, then there are precisely three generating sets $\{1,4\},\{2,3\}$ and $\{1,2,3,4\}$. If we take $n=5$ in Theorem 2.1, then we get $|N(S)|=3$.

Theorem 2.3. Let $\Gamma \cong \mathbb{Z}_{2}^{t} \times \mathbb{Z}_{2 n}$. Then the number of generating sets consisting of only nonzero self-inverse elements are $2^{\left(2^{t+1}-1\right)}-1$.

Proof. Let us consider $\Gamma \cong \mathbb{Z}_{2}^{t} \times \mathbb{Z}_{2 n}$ and our aim is to show that number of generating sets consisting of only self-inverse elements are $2^{\left(2^{t+1}-1\right)}-1$. Note that 
each element of $\mathbb{Z}_{2}^{t}$ is self inverse and there are precisely two self inverse elements namely ' 0 ' and ' $n$ ' in $\mathbb{Z}_{2 n}$. By fixing exactly one from ' 0 ' and ' $n$ ' at $(t+1)^{t h}$ position all self inverse elements can be obtained. Firstly, by fixing zero at $(t+1)^{t h}$ position in $(t+1)$ tuple, i.e. $\left(a_{1}, a_{2}, \ldots, a_{t}, 0\right)$, there are $2^{t}-1$ choices for $a_{i}$ and hence $2^{t}-1$ nonzero self inverse elements. In the similar way, by fixing ' $n$ ' at $(t+1)^{t h}$ position in $(t+1)$ tuple, i.e., $\left(a_{1}, a_{2}, \ldots, a_{t}, n\right)$ there are $2^{t}$ choices for $a_{i}$ and hence for self inverse elements. Therefore the total number of non-zero self inverse elements in $\Gamma$ are $2^{t+1}-1$. Now it is easy to see that the number of generating sets consisting of only self-inverse elements are $2^{\left(2^{t+1}-1\right)}-1$.

Remark 2.4. Let $\Gamma \cong \mathbb{Z}_{2} \times \mathbb{Z}_{2 n}$. Then there are exactly three nonzero self-inverse elements and hence six generating set consisting of nonzero self-inverse elements.

\section{Cayley Graphs and Algebraic Graphs}

Let $(A, *)$ be an algebraic structure. A graph $G:=(V, E)$ is called an algebraic graph, if $V \subseteq A$ and the adjacency rule is due to binary operation ' $*$ ' of algebraic structure. In this section, we establish the results that gives the relation between Cayley graph and algebraic graphs, namely, unit graph, co-unit graph, total graphs and co-total graphs.

Theorem 3.1. Let $\tilde{G}\left(\mathbb{Z}_{n}\right)$ and $\tilde{T}\left(\Gamma\left(\mathbb{Z}_{n}\right)\right)$ be co-unit graph, and co-total graph, respectively. Then for a positive integer ' $n$ ' the following holds:

(1) $\operatorname{Cay}\left(\mathbb{Z}_{n}, S\right) \cong \tilde{G}\left(\mathbb{Z}_{n}\right)$, when $S=U\left(\mathbb{Z}_{n}\right)$;

(2) $\operatorname{Cay}\left(\mathbb{Z}_{n}, S\right) \cong \tilde{T}\left(\Gamma\left(\mathbb{Z}_{n}\right)\right)$, when $S=Z^{0}\left(\mathbb{Z}_{n}\right)$.

Proof. It is clear that the vertex set of both $\operatorname{Cay}\left(\mathbb{Z}_{n}, S\right)$ and $\tilde{G}\left(\mathbb{Z}_{n}\right)$ are same. Let $v_{i}, v_{j} \in V\left(\operatorname{Cay}\left(\mathbb{Z}_{n}, S\right)\right)$. Then $v_{i}$ is adjacent to $v_{j}$ if and only if $v_{i}-v_{j} \in S$.

First, when $S=U\left(\mathbb{Z}_{n}\right)$, this implies that $v_{i}-v_{j} \in U\left(\mathbb{Z}_{n}\right)$. This shows that $\left(v_{i}, v_{j}\right) \in E\left(\operatorname{Cay}\left(\mathbb{Z}_{n}, S\right)\right)$ if and only if $\left(v_{i}, v_{j}\right) \in E\left(\tilde{G}\left(\mathbb{Z}_{n}\right)\right)$. Therefore, $\operatorname{Cay}\left(\mathbb{Z}_{n}, S\right) \cong \tilde{G}\left(\mathbb{Z}_{n}\right)$.

On the other hand, when $S=Z^{0}\left(\mathbb{Z}_{n}\right)$. This implies that $v_{i}-v_{j} \in Z^{0}\left(\mathbb{Z}_{n}\right)$. This shows that $\left(v_{i}, v_{j}\right) \in E\left(\operatorname{Cay}\left(\mathbb{Z}_{n}, S\right)\right)$ if and only if $\left(v_{i}, v_{j}\right) \in E\left(\tilde{T}\left(\Gamma\left(\mathbb{Z}_{n}\right)\right)\right)$. Therefore, $\operatorname{Cay}\left(\mathbb{Z}_{n}, S\right) \cong \tilde{T}\left(\Gamma\left(\mathbb{Z}_{n}\right)\right)$.

Lemma 3.2. Let $n$ be an even positive integer. Then for all $a, b \in \mathbb{Z}_{n}$ the following holds:

(i) For $S=U\left(\mathbb{Z}_{n}\right) ; a-b \in S$ if and only if $a+b \in S$;

(ii) For $S=Z^{0}\left(\mathbb{Z}_{n}\right) ; a-b \in S$ if and only if $a+b \in S \cup\{0\}$.

Proof. For the case $(i) ;(\Rightarrow)$ Let $n$ be an even positive integer and $S=U\left(\mathbb{Z}_{n}\right)$. Then $U\left(\mathbb{Z}_{n}\right)$ consists of all odd positive integer less than $n$. If $a-b \in S=U\left(\mathbb{Z}_{n}\right)$, then $a-b$ must be an odd number. Note that $a+b=a-b+2 b$, which is an odd number, and hence $a+b \in S$.

$(\Leftarrow)$ If $a+b \in S=U\left(\mathbb{Z}_{n}\right)$, then $a+b$ must be an odd number. Note that in this 
case exactly one $a$ or $b$ must be odd and hence $a-b$ is an odd number. Therefore $a-b \in S$.

For the case $(i i) ;(\Rightarrow)$ if $S=Z^{0}\left(\mathbb{Z}_{n}\right)$. Then $Z^{0}\left(\mathbb{Z}_{n}\right)$ consists of all even positive integer less than $n$. If $a-b \in S=Z^{0}\left(\mathbb{Z}_{n}\right)$, then $a-b$ must be an even number. Note that $a+b=a-b+2 b$, which is an even number may be zero, and hence $a+b \in S \cup\{0\}$.

$(\Leftarrow)$ If $a+b \in S=Z^{0}\left(\mathbb{Z}_{n}\right) \cup\{0\}$, then $a+b$ must be an even number. Note that in this case either both $a$ and $b$ are even or both are odd. Then $a-b$ is an even number, and hence $a-b \in S$.

Theorem 3.3. Let $G\left(\mathbb{Z}_{n}\right)$ and $T\left(\Gamma\left(G\left(\mathbb{Z}_{n}\right)\right)\right)$ be unit graph and total graph, respectively. Then for an even positive integer ' $n$ ' the following holds:

(1) $\operatorname{Cay}\left(\mathbb{Z}_{n}, S\right) \cong G\left(\mathbb{Z}_{n}\right)$, when $S=U\left(\mathbb{Z}_{n}\right)$;

(2) $\operatorname{Cay}\left(\mathbb{Z}_{n}, S\right) \cong T\left(\Gamma\left(\mathbb{Z}_{n}\right)\right)$, when $S=Z^{0}\left(\mathbb{Z}_{n}\right)$.

Proof. To show the result it is suffices to show the isomorphism between the given graphs. Let ' $n$ ' be an even positive integer. To tackle the case (1), define a function $f: V\left(\operatorname{Cay}\left(\mathbb{Z}_{n}, S\right)\right) \rightarrow V\left(G\left(\mathbb{Z}_{n}\right)\right)$ such that

$$
f(v)= \begin{cases}v, & \text { if } v \text { is odd } \\ n-v, & \text { otherwise. }\end{cases}
$$

Clearly $f$ is a bijective function. Now we want to show that under this $f$ adjacency is preserved. To do this, let $v_{i}$ and $v_{j}$ be two vertices of $\operatorname{Cay}\left(\mathbb{Z}_{n}, S\right)$. Then $v_{i}$ is adjacent to $v_{j}$, if $v_{i}-v_{j} \in S=U\left(\mathbb{Z}_{n}\right)$ which is odd and this is possible only when either both $v_{i}, v_{j} \in Z^{0}\left(\mathbb{Z}_{n}\right)$ or exactly one of them $v_{i}$ or $v_{j}$ belong to $U\left(\mathbb{Z}_{n}\right)$.

For the first possibility, one of $v_{i}$ or $v_{j}$ must be odd although belong to $Z^{0}\left(\mathbb{Z}_{n}\right)$, then $f\left(v_{i}\right)=v_{i}$ and $f\left(v_{j}\right)=n-v_{j}$, this implies that $f\left(v_{i}\right)+f\left(v_{j}\right)=v_{i}+n-v_{j}=$ $n+\left(v_{i}-v_{j}\right) \in U\left(\mathbb{Z}_{n}\right)$. Next, if $v_{i} \in U\left(\mathbb{Z}_{n}\right)$ and $v_{j} \in Z^{0}\left(\mathbb{Z}_{n}\right)$, then $f\left(v_{i}\right)=v_{i}$ and $f\left(v_{j}\right)=n-v_{j}$ and the sum $f\left(v_{i}\right)+f\left(v_{j}\right)=v_{i}+n-v_{j}=n+\left(v_{i}-v_{j}\right) \in U\left(\mathbb{Z}_{n}\right)$. This implies that $f\left(v_{i}\right)+f\left(v_{j}\right) \in U\left(\mathbb{Z}_{n}\right)$. This shows that $\left(v_{i}, v_{j}\right) \in E\left(\operatorname{Cay}\left(\mathbb{Z}_{n}, S\right)\right) \Leftrightarrow$ $\left(f\left(v_{i}\right), f\left(v_{j}\right)\right) \in E\left(G\left(\mathbb{Z}_{n}\right)\right)$. Therefore $\operatorname{Cay}\left(\mathbb{Z}_{n}, S\right) \cong G\left(\mathbb{Z}_{n}\right)$, when $S=U\left(\mathbb{Z}_{n}\right)$.

For the case (2), again define a function $f: V\left(\operatorname{Cay}\left(\mathbb{Z}_{n}, S\right)\right) \rightarrow V\left(T\left(\Gamma\left(\mathbb{Z}_{n}\right)\right)\right)$ such that

$$
f(v)= \begin{cases}v, & \text { if } v \text { is even } \\ n-v, & \text { otherwise. }\end{cases}
$$

Clearly $f$ is a bijective function. Now we want to show that under this $f$ adjacency is preserved. To do this, let $v_{i}$ and $v_{j}$ be two vertices of $\operatorname{Cay}\left(\mathbb{Z}_{n}, S\right)$. Then $v_{i}$ is adjacent to $v_{j}$, if $v_{i}-v_{j} \in S=Z^{0}\left(\mathbb{Z}_{n}\right)$. Then there are two possibilities, either $v_{i}-v_{j}$ is even or odd.

First, if $v_{i}-v_{j}$ is even, then either both $v_{i}, v_{j}$ are even or odd. Let us suppose both $v_{i}$ and $v_{j}$ are even. Then the sum $f\left(v_{i}\right)+f\left(v_{j}\right)=v_{i}+v_{j}$ which is even and belong to $Z\left(\mathbb{Z}_{n}\right)$ (using Lemma 3.2). Now, if both $v_{i}, v_{j}$ are odd, then the sum $f\left(v_{i}\right)+f\left(v_{j}\right)=\left(n-v_{i}\right)+\left(n-v_{j}\right)=2 n-\left(v_{i}+v_{j}\right)$ again belong to $Z\left(\mathbb{Z}_{n}\right)$. Hence in the first possibility, $v_{i}-v_{j} \in Z^{0}\left(\mathbb{Z}_{n}\right)$ if and only if $f\left(v_{i}\right)+f\left(v_{j}\right) \in Z\left(\mathbb{Z}_{n}\right)$. Next, in the second possibility, if $v_{i}-v_{j} \in Z^{0}\left(\mathbb{Z}_{n}\right)$ and it is odd, then note that at least one 
of $v_{i}$ or $v_{j}$ must be odd. Let us suppose $v_{i}$ be odd. Then the sum $f\left(v_{i}\right)+f\left(v_{j}\right)=$ $\left(n-v_{i}\right)+v_{j}=n-\left(v_{i}-v_{j}\right)$. Consequently, $f\left(v_{i}\right)+f\left(v_{j}\right) \in Z\left(\mathbb{Z}_{n}\right)$. Hence in both the possibilities adjacency is preserved under $f$. Therefore $\operatorname{Cay}\left(\mathbb{Z}_{n}, S\right) \cong T\left(\Gamma\left(\mathbb{Z}_{n}\right)\right)$, when $S=Z^{0}\left(\mathbb{Z}_{n}\right)$.

Remark 3.4. It may hence be observed from Theorem 3.3 that the study on the notion of unit graph and total graph for $\mathbb{Z}_{n}$ is a particular case for the study on the $\operatorname{Cay}\left(\mathbb{Z}_{n}, S\right)$ associated with specify generating sets, viz., $U\left(\mathbb{Z}_{n}\right)$ and $Z^{0}\left(\mathbb{Z}_{n}\right)$.

Theorem 3.5. If $S_{1} \subseteq S_{2}$, then Cay $\left(\mathbb{Z}_{n}, S_{1}\right)$ is a subgraph of Cay $\left(\mathbb{Z}_{n}, S_{2}\right)$.

Proof. Let $S_{1} \subseteq S_{2}$. Clearly, the vertex set of $\operatorname{Cay}\left(\mathbb{Z}_{n}, S_{1}\right)$ and $\operatorname{Cay}\left(\mathbb{Z}_{n}, S_{2}\right)$ is same and is equal to $\{0,1,2, \ldots, n-1\}$. Let $v_{i}$ and $v_{j}$ be two arbitrary vertices in $\operatorname{Cay}\left(\mathbb{Z}_{n}, S_{1}\right)$. If $v_{i}$ and $v_{j}$ are adjacent in $\operatorname{Cay}\left(\mathbb{Z}_{n}, S_{1}\right)$, then $v_{i}-v_{j} \in S_{1}$. This implies that $v_{i}-v_{j} \in S_{2}$ as $S_{1} \subseteq S_{2}$. Therefore each edge of $\operatorname{Cay}\left(\mathbb{Z}_{n}, S_{1}\right)$ is also an edge of $\operatorname{Cay}\left(\mathbb{Z}_{n}, S_{2}\right)$. Hence, $\operatorname{Cay}\left(\mathbb{Z}_{n}, S_{1}\right)$ is a subgraph of $\operatorname{Cay}\left(\mathbb{Z}_{n}, S_{2}\right)$.

Theorem 3.6. Let $\Gamma \cong \mathbb{Z}_{2}^{t} \times \mathbb{Z}_{2 n}$ and $S$ be the collection of all self inverse elements of $\Gamma$.

(1) If $S^{\prime} \subseteq S$ and $\left|S^{\prime}\right|=1$, then $\operatorname{Cay}\left(\Gamma, S^{\prime}\right) \cong \underbrace{K_{2} \cup K_{2} \cup \cdots \cup K_{2}}_{\left(2^{t} n\right)-\text { times }}$;

(2) If $S^{\prime}=S$, then $\operatorname{Cay}(\Gamma, S) \cong \underbrace{K_{|S+1|} \cup K_{|S+1|} \cup \cdots \cup K_{|S+1|}}_{n-\text { times }}$.

Proof. Case (1): Let $S^{\prime} \subseteq S$ and $\left|S^{\prime}\right|=1$. Then $\operatorname{Cay}\left(\Gamma, S^{\prime}\right)$ is 1-regular graph. The only possible 1-regular graph is either $K_{2}$ or copies of $K_{2}$. Since there are $2^{t+1} . n$ vertices in $\operatorname{Cay}\left(\Gamma, S^{\prime}\right)$, this indicates that $\operatorname{Cay}\left(\Gamma, S^{\prime}\right)$ is isomorphic to copies of $K_{2}$. Also to form $K_{2}$ only two vertices are required. Hence, there are $2^{t} \cdot n$ copies of $K_{2}$. Therefore $\operatorname{Cay}\left(\Gamma, S^{\prime}\right) \cong \underbrace{K_{2} \cup K_{2} \cup \cdots \cup K_{2}}_{\left(2^{t} n\right) \text {-times }}$.

Case (2): From the Theorem 2.3 clearly, $\left(2^{t+1}-1\right)$ non-zero self inverse elements in $\Gamma$. This implies that $|S|=2^{(t+1)}-1$. Then, $\operatorname{Cay}(\Gamma, S)$ is $2^{(t+1)}-1$ regular graph. Let $V^{\prime}=\left\{\left(a_{1}, a_{2}, \ldots, a_{t}, a_{t+1}\right): a_{i} \in\{0,1\}(1 \leq i \leq t)\right.$ and $a_{t+1} \in$ $\{0, n\}\}$. The set $V^{\prime}$ is subset of $V(\operatorname{Cay}(\Gamma, S))$ and specifically it is the collection of all self inverse elements, i.e., $V^{\prime}=S \cup(0,0, \ldots, 0,0)$. Let $v_{i}$ and $v_{j}$ be two arbitrary vertices of $V^{\prime}$. Then $v_{i}-v_{j}=\left(c_{1}, c_{2}, \ldots, c_{t}, c_{t+1}\right) \backslash(0,0, \ldots, 0): c_{i} \in$ $\{0,1\}(1 \leq i \leq t)$ and $c_{t+1} \in\{0, n\}$. This implies that $v_{i}-v_{j} \in S$, also $v_{i}$ and $v_{j}$ are arbitrary which indicates that for all $v_{i}, v_{j} \in V^{\prime}, v_{i}-v_{j} \in S$. Therefore all the vertices of $V^{\prime}$ are mutually adjacent and each of them have degree $2^{(t+1)}-1$. Also no vertex of $V^{\prime}$ is adjacent to other vertices of $\operatorname{Cay}(\Gamma, S)$ as $\operatorname{Cay}(\Gamma, S)$ is $2^{(t+1)}-1$ regular. Let $V^{\prime \prime}=\left\{\left(a_{1}, a_{2}, \ldots, a_{t}, a_{t+1}+1\right): a_{i} \in\{0,1\}(1 \leq i \leq\right.$ $t)$ and $\left.a_{t+1} \in\{0, n\}\right\}$. Let $v_{i}^{\prime}$ and $v_{j}^{\prime}$ be two arbitrary vertices of $V^{\prime \prime}$. Then $v_{i}^{\prime}-v_{j}^{\prime}=\left(d_{1}, d_{2}, \ldots, d_{t}, d_{t+1}\right) \backslash(0,0,, \ldots, 0,0): d_{i} \in\{0,1\}(1 \leq i \leq t)$ and $d_{t+1} \in$ $\{0, n\}$. This implies that $v_{i}^{\prime}-v_{j}^{\prime} \in S$, also $v_{i}^{\prime}$ and $v_{j}^{\prime}$ are arbitrary which indicates that for all $v_{i}^{\prime}, v_{j}^{\prime} \in V^{\prime \prime}, v_{i}^{\prime}-v_{j}^{\prime} \in S$. Therefore all the vertices of $V^{\prime \prime}$ are again 
mutually adjacent and each of them have degree $2^{(t+1)}-1$. Also no vertex of $V^{\prime \prime}$ is adjacent to other vertices of $\operatorname{Cay}(\Gamma, S)$ as $\operatorname{Cay}(\Gamma, S)$ is $2^{(t+1)}-1$ regular. Following the same procedure, one get the copies of $K_{2^{t+1}}$. Since there are $n .2^{(t+1)}$ vertices in $\operatorname{Cay}(\Gamma, S)$, so there will be precisely $n$ copies of $K_{2^{t+1}}$ are obtained. Therefore, $\operatorname{Cay}(\Gamma, S) \cong \underbrace{K_{|S+1|} \cup K_{|S+1|} \cup \cdots \cup K_{|S+1|}}_{n \text {-times }}$.

Corollary 3.7. Let $\Gamma \cong \mathbb{Z}_{2}^{t}$ and $S$ be the collection of all self inverse elements of $\Gamma$. Then the followings holds:

(1) If $S^{\prime} \subseteq S$ and $\left|S^{\prime}\right|=1$, then $\operatorname{Cay}\left(\Gamma, S^{\prime}\right) \cong \underbrace{K_{2} \cup K_{2} \cup \cdots \cup K_{2}}_{\left(2^{t-1}\right)-\text { times }}$;

(2) If $S^{\prime}=S$, then $\operatorname{Cay}\left(\Gamma, S^{\prime}\right) \cong K_{2^{t}}$.

Proof. By taking $n=1$ in Theorem 3.6, the proof follows.

Theorem 3.8. Let $\Gamma \cong \mathbb{Z}_{2}^{t} \times \mathbb{Z}_{2 n}$ and $S$ be the collection of all self inverse elements of $\Gamma$. Then for $n=1, \operatorname{Cay}(\Gamma, S)$ is connected.

Proof. Invoking Theorem 3.6 and Corollary 3.7, $\operatorname{Cay}\left(\Gamma, S^{\prime}\right) \cong K_{2^{t+1}}$, and hence connected.

Theorem 3.9. Let $S=\left\{\left(1,1, \ldots, 1, a_{t}\right), a_{t} \in U\left(\mathbb{Z}_{2 n}\right)\right\}$. Then $\operatorname{Cay}\left(\mathbb{Z}_{2}^{t} \times \mathbb{Z}_{2 n}, S\right) \cong$ $G\left(\mathbb{Z}_{2}^{t} \times \mathbb{Z}_{2 n}\right) \cong \tilde{G}\left(\mathbb{Z}_{2}^{t} \times \mathbb{Z}_{2 n}\right)$.

Proof. Let us consider $\mathbb{Z}_{2 n}$ and we want to show that $\operatorname{Cay}\left(\mathbb{Z}_{2}^{t} \times \mathbb{Z}_{2 n}, S\right) \cong G\left(\mathbb{Z}_{2}^{t} \times\right.$ $\left.\mathbb{Z}_{2 n}\right) \cong \tilde{G}\left(\mathbb{Z}_{2}^{t} \times \mathbb{Z}_{2 n}\right)$. For this first we will show $\operatorname{Cay}\left(\mathbb{Z}_{2}^{t} \times \mathbb{Z}_{2 n}, S\right) \cong G\left(\mathbb{Z}_{2}^{t} \times \mathbb{Z}_{2 n}\right)$ and then $\operatorname{Cay}\left(\mathbb{Z}_{2}^{t} \times \mathbb{Z}_{2 n}, S\right) \cong \tilde{G}\left(\mathbb{Z}_{2}^{t} \times \mathbb{Z}_{2 n}\right)$. To do this let us suppose on contrary that

$$
\operatorname{Cay}\left(\mathbb{Z}_{2}^{t} \times \mathbb{Z}_{2 n}, S\right) \nsucceq G\left(\mathbb{Z}_{2}^{t} \times \mathbb{Z}_{2 n}\right)
$$

Although $V\left(\operatorname{Cay}\left(\mathbb{Z}_{2}^{t} \times \mathbb{Z}_{2 n}, S\right)\right)=V\left(G\left(\mathbb{Z}_{2}^{t} \times \mathbb{Z}_{2 n}\right)\right)$. Let $v_{i}=\left(a_{1}, a_{2}, \ldots, a_{t}, a_{t+1}\right)$ and $v_{j}=\left(b_{1}, b_{2}, \ldots, b_{t}, b_{t+1}\right)$ be two vertices of $\operatorname{Cay}\left(\mathbb{Z}_{2}^{t} \times \mathbb{Z}_{2 n}, S\right)$. Then $v_{i}$ and $v_{j}$ are adjacent in $\operatorname{Cay}\left(\mathbb{Z}_{2}^{t} \times \mathbb{Z}_{2 n}, S\right)$ whenever $v_{i}-v_{j} \in S$, i.e., $\left(a_{1}-b_{1}, a_{2}-\right.$ $\left.b_{2}, \ldots, a_{t}-b_{t}, a_{t+1}-b_{t+1}\right), a_{i}-b_{i}=1$ for all $1 \leq i \leq t$ and $a_{t+1}-b_{t+1} \in U\left(\mathbb{Z}_{2 n}\right)$. Since $a_{i}, b_{i} \in \mathbb{Z}_{2}, a_{i}-b_{i}=1$ gives us that one of $a_{i}$ or $b_{i}$ must be ' 0 '. This implies that $a_{i}+b_{i}=1$ for all $1 \leq i \leq t$. As $a_{t+1}-b_{t+1} \in U\left(\mathbb{Z}_{2 n}\right)$, this implies that $a_{t+1}+b_{t+1} \in U\left(\mathbb{Z}_{2 n}\right)$. Hence $\left(v_{i}, v_{j}\right) \in E\left(\operatorname{Cay}\left(\mathbb{Z}_{2}^{t} \times \mathbb{Z}_{2 n}, S\right)\right)$. This implies that $\left(v_{i}, v_{j}\right) \in E\left(G\left(\mathbb{Z}_{2}^{t} \times \mathbb{Z}_{2 n}\right)\right)$. This shows that each edge of $\operatorname{Cay}\left(\mathbb{Z}_{2}^{t} \times \mathbb{Z}_{2 n}, S\right)$ is also an edge of $G\left(\mathbb{Z}_{2}^{t} \times \mathbb{Z}_{2 n}\right)$. Therefore only possibility is to be (1) is that two vertices $v_{i}^{\prime}$ $v_{j}^{\prime}$ are adjacent in $G\left(\mathbb{Z}_{2}^{t} \times \mathbb{Z}_{2 n}\right)$ but not in $\operatorname{Cay}\left(\mathbb{Z}_{2}^{t} \times \mathbb{Z}_{2 n}, S\right)$, i.e., $v_{i}^{\prime}+v_{j}^{\prime} \in U\left(\mathbb{Z}_{2 n}\right)$, but $v_{i}^{\prime}-v_{j}^{\prime} \notin S$. From Lemma 3.2, $v_{i}^{\prime}+v_{j}^{\prime} \in U\left(\mathbb{Z}_{2 n}\right)$ implies that $v_{i}^{\prime}-v_{j}^{\prime} \in U\left(\mathbb{Z}_{2 n}\right)$. This gives us $S \subset U\left(\mathbb{Z}_{2}^{t} \times \mathbb{Z}_{2 n}\right)$, a contradiction. Hence our assumption is wrong. Therefore $\operatorname{Cay}\left(\mathbb{Z}_{2}^{t} \times \mathbb{Z}_{2 n}, S\right) \cong G\left(\mathbb{Z}_{2}^{t} \times \mathbb{Z}_{2 n}\right)$.

Now to show that $\operatorname{Cay}\left(\mathbb{Z}_{2}^{t} \times \mathbb{Z}_{2 n}, S\right) \cong \tilde{G}\left(\mathbb{Z}_{2}^{t} \times \mathbb{Z}_{2 n}\right)$. Let $v_{i}=\left(a_{1}, a_{2}, \ldots\right.$, $\left.a_{t}, a_{t+1}\right)$ and $v_{j}=\left(b_{1}, b_{2}, \ldots, b_{t}, b_{t+1}\right)$ be two vertices of $\operatorname{Cay}\left(\mathbb{Z}_{2}^{t} \times \mathbb{Z}_{2 n}, S\right)$, although $V\left(\operatorname{Cay}\left(\mathbb{Z}_{2}^{t} \times \mathbb{Z}_{2 n}, S\right)\right)=V\left(\tilde{G}\left(\mathbb{Z}_{2}^{t} \times \mathbb{Z}_{2 n}\right)\right)$. Then $v_{i}$ and $v_{j}$ are adjacent in $\operatorname{Cay}\left(\mathbb{Z}_{2}^{t} \times\right.$ 
$\left.\mathbb{Z}_{2 n}, S\right)$ whenever $v_{i}-v_{j} \in S$. Since $S=\left\{\left(1,1, \ldots, 1, a_{k}\right), a_{k} \in U\left(\mathbb{Z}_{2 n}\right)\right\}$, which indicates that $\left(v_{i}, v_{j}\right) \in E\left(\operatorname{Cay}\left(\mathbb{Z}_{2}^{t} \times \mathbb{Z}_{2 n}, S\right)\right)$ if and only if $\left(v_{i}, v_{j}\right) \in E\left(\tilde{G}\left(\mathbb{Z}_{2}^{t} \times \mathbb{Z}_{2 n}\right)\right)$. This shows that each edge of $\operatorname{Cay}\left(\mathbb{Z}_{2}^{t} \times \mathbb{Z}_{2 n}, S\right)$ is also an edge of $\tilde{G}\left(\mathbb{Z}_{2}^{t} \times \mathbb{Z}_{2 n}\right)$. Therefore $\operatorname{Cay}\left(\mathbb{Z}_{2}^{t} \times \mathbb{Z}_{2 n}, S\right) \cong \tilde{G}\left(\mathbb{Z}_{2}^{t} \times \mathbb{Z}_{2 n}\right)$.

Theorem 3.10. Let $S=\left\{\left(a_{1}, a_{2}, \ldots, a_{t-1}, a_{t}\right)\right\}$, with either at least one $a_{i}=0$ for $1 \leq i \leq t-1$ and $a_{t} \in \mathbb{Z}_{2 n} \backslash\{0\}$ or $a_{i}=1$ for all $i, 1 \leq i \leq t-1$ and $a_{t} \in Z\left(\mathbb{Z}_{2 n}\right) \cup\{0\}$. Then $\operatorname{Cay}\left(\mathbb{Z}_{2}^{t} \times \mathbb{Z}_{2 n}, S\right) \cong T\left(\Gamma\left(\mathbb{Z}_{2}^{t} \times \mathbb{Z}_{2 n}\right)\right) \cong \tilde{T}\left(\Gamma\left(\mathbb{Z}_{2}^{t} \times \mathbb{Z}_{2 n}\right)\right)$.

Proof. The proof can be obtained by the arguments that are analogous to those given in the proof of Theorem 3.9.

Acknowledgement. This paper is dedicated to Prof. A.D.N. Bajpai (Great academician, excellent poet and very polite personality of Rani Durgavati University, Jabalpur, M.P., India) on the occasion of his coming 65th birthday.

\section{REFERENCES}

[1] Beny, G. A. and Rakhmonov, Z., Number of Cayley graph of finite group $Z_{n}$ that is undirected, Proceedings of third Conference and Workshop on Group theory, University of Tehran, 9-10 March (2011), 8-11.

[2] Cayley, A., Desiderata and suggestions; The Theory of groups: graphical representation, American Journal of Mathematics, 1(2) (1878), 174-176.

[3] Harary, F., Graph Theory, Addison-Wesley Publ. Comp. Reading. MA, 1969.

[4] Jacobson, N., Lectures in Abstract Algebra, East-West Press P. Ltd., New Delhi, 1951.

[5] Maimani, H. R., Pournaki, M. R., Tehranian, A. and Yassemi, S. Graphs Attached to Rings Revisited, Arabian Journal for Science and Engineering (2011), 36:997. 